\title{
Advances in random lasing sensing
}

Federico Tommasi, Emilio Ignesti, Lorenzo Fini, Fabrizio Martelli, Stefano Cavalieri

Federico Tommasi, Emilio Ignesti, Lorenzo Fini, Fabrizio Martelli, Stefano Cavalieri, "Advances in random lasing sensing," Proc. SPIE 11028, Optical Sensors 2019, 110280L (11 April 2019); doi: 10.1117/12.2522329

SPIE. Event: SPIE Optics + Optoelectronics, 2019, Prague, Czech Republic 


\title{
Advances in random lasing sensing
}

\author{
Federico Tommasi, Emilio Ignesti, Lorenzo Fini, Fabrizio Martelli, and Stefano Cavalieri \\ Dipartimento di Fisica e Astronomia, Università di Firenze, \\ via G. Sansone 1, I-50019 Sesto Fiorentino, Italy
}

\begin{abstract}
A random laser is an optical system where the light is amplified by stimulated emission along random paths in a disordered medium. In recent years, a new kind of non-invasive sensor based on random lasing has been proposed. ${ }^{1,2}$ The striking point is that a sensor based on random lasing has an emission "fed" by the feedback due to the scattering properties of the medium, making such a system a natural candidate for studying materials with strong disorder. Here, we report the recent advances in the sensor structure and performances.
\end{abstract}

Keywords: random laser, optical sensor

\section{INTRODUCTION}

Random lasing is an optical phenomenon that can take place inside a disordered medium where a pumping system establishes a population inversion. The stimulated emission mechanism amplifies the optical radiation along paths sustained by random scattering events instead by an optical cavity. ${ }^{3-14}$ Such a "laser-like" radiation exhibits a spectral narrowing, without a fixed direction and the presence of speckles. In general, a random laser system is composed by a mixture of an active medium, such as laser dye $\mathrm{e}^{15}$ or powder of doped crystal for solid state laser, ${ }^{16}$ and scattering material, such as nanoparticles or disorder induced in the structure of the bulk material. The pumping system is often provided by a laser beam. Random lasing has been observed in biological material ${ }^{17-23}$ and differences between healthy and cancerous human tissue has also been reported. ${ }^{24-28}$

Developing a sensor based on random lasing ${ }^{29-32}$ is of great interest, since random laser has an emission "fed" the feedback due to the scattering, making such a system a natural candidate for studying materials with strong disorder. However, the progresses in biological applications have been slowed by the requirement to the directly irradiate the sample by the pumping system and to infiltrate the biological tissue with toxic gain material, although biocompatible suitable substances have been proposed to mitigate the latter problem ${ }^{33,34}$

In recent years, ${ }^{1,2}$ a new kind of non invasive sensor based on random lasing has been proposed; such a sensor consists in a random laser system put inside a spherical transparent box able to optically communicate with an external sensed medium. The system is maintained below threshold until it is put in contact with a scattering external medium, so guarantying a feedback signal amplified by stimulated emission. Moreover, the physical separation between the sensed medium and the material inside the sensor guarantees the non invasivity, whereas the optical fiber coupling allows the portability as well. Here, we report the recent advances in the sensor structure and performances, in particular in sensing the dimension of scattering particles. In particular, we have found that, for a wide range of different diameters of scattering particles dispersed in an external medium, the peak intensity of the emission spectrum only depends on the reduced scattering coefficient, whereas the other signal features can be analyzed to retrieve information about the particles sizes.

\section{SENSOR STRUCTURE}

The sensor consists in a glass hollow sphere with an active medium inside. Through the glass, the sensor is able to communicate with the external disordered medium through the emitted and received light. Indeed, spontaneously emitted radiation can propagate to the external disordered medium and then, after a random path guided by diffusion, an amount of light can go back to the sphere, thus undergoing amplification by stimulated emission in the active medium. Hence, the external disordered medium provides a feedback mechanism for the spontaneously emitted radiation, in a way dependent on the characteristics of the disordered external material.

Optical Sensors 2019, edited by Francesco Baldini, Jiri Homola, Robert A. Lieberman, Proc. of SPIE

Vol. 11028, 110280L · C 2019 SPIE · CCC code: 0277-786X/19/\$18 · doi: 10.1117/12.2522329 


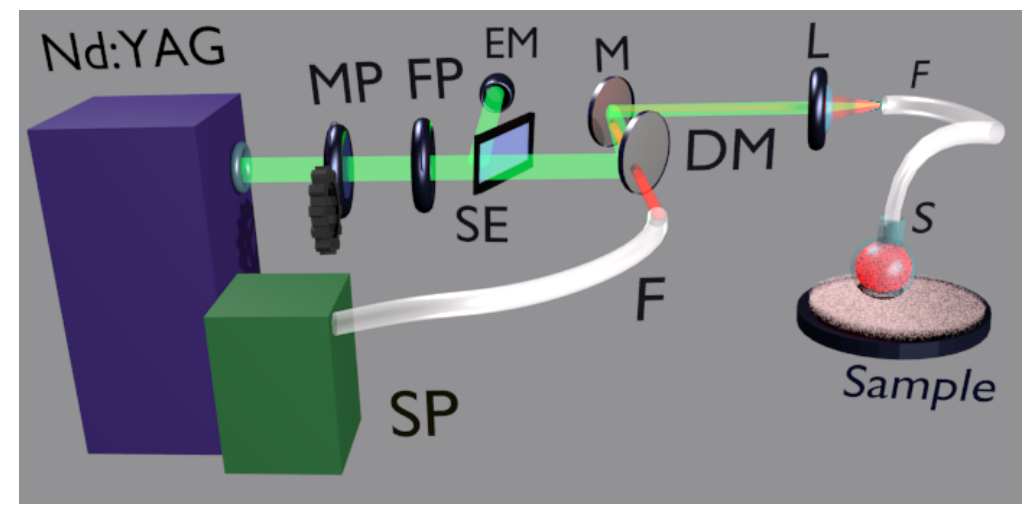

Figure 1. Experimental set-up scheme: $\mathrm{SP}=$ spectrometer, $\mathrm{MP}=$ movable polarizer, $\mathrm{FP}=$ fixed polarizer, $\mathrm{SE}=$ semitransparent plate, $\mathrm{EM}=$ energy meter, $\mathrm{M}=$ mirror, $\mathrm{DM}=$ dichroic mirror, $\mathrm{F}=$ fiber, $\mathrm{L}=$ lens, $\mathrm{S}=$ sample. The green beam is the pump and the red one is the signal. Among the DM and the S the two beam are overlapped.

The active medium inside the cell consists in an alcoholic solution of dye with a small amount of nanoparticles added to prevent laser action due the refractive index mismatch between the solution and the glass. The pump beam is provided by a Q-switched Nd:YAG laser and the output signal is sent to the spectrometer by an optical fiber that has an extremity inside the sensor. By using a dichroic mirror the pump beam is injected into the same fiber that carries the signal.

The pumping energy is tuned by a pair of polarizers: the first movable by a stepper motor connected to a PC and the second fixed. A reflection from a semi-transparent plate is sent to an energy meter to indirectly measure the pumping energy. An automatic acquisition system allows to store the spectrum of each signal pulse and the energy of the corresponding pump pulse.

The diffusive samples are put in a square container with black walls of side $1.0 \mathrm{~cm}$. The dimensions of the scattering cell are wide enough to avoid wall effects, for the used particles concentrations. The sensor is partially immersed inside the liquid sample at the same fixed depth (for the half of the sensor diameter) and is positioned at the center of the cross section of the cubic cell. In Fig. 1 a scheme of the experimental set-up is shown. The signal shows an over-threshold behavior with a spectral narrowing once the back scattering from the disordered external medium becomes strong enough to provided a sufficient feedback. Hence, the spectral characteristics are strictly connected to the scattering properties, given by the particles size, concentration and refractive index, of the sensed sample.

\section{MEASUREMENT METHOD}

In this section, a method to directly measure the scattering property of a water dispersion of nanoparticles of different dimensions is reported. The method core makes use of a dilution of Intralipid $20 \%$, an emulsion of fat particles suspended in water, as reference sample. Indeed, such a medium has a large characterization and stability ${ }^{35-41}$ and the scattering coefficient $\mu_{s}$ and the reduce scattering coefficient $\mu_{s}^{\prime}=\mu_{s}(1-g)$, where $g$ is the asymmetry factor of the scattering function, can be a priori known as the dilution varies. Then, the reference sample has been prepared with $\mu_{s}^{\prime}$ of $2.33 \mathrm{~mm}^{-1}$ at the radiation wavelength of the signal, that corresponds to $0.430 \mathrm{~mm}$ of transport mean free path, a value typical of diffusive media such as biological tissues. The absorption coefficient $\mu_{a}$ is very small and almost identical to that of the water and its contribute can be here neglected.

Following the hypotheses that $\mu_{s}^{\prime}$ is the main parameters that determines the scattering feedback to the sensor, samples composed by water monodispersion of nanoparticles of different size are prepared, varying the concentration in order to reach the same $\mu_{s}^{\prime}$ of the reference. By using the particles radius distribution provided by the manufacturers of the particles, the desired concentration can be a priori calculated by the single scattering

Send correspondence to Federico Tommasi: E-mail: federico.tommasi@unifi.it 


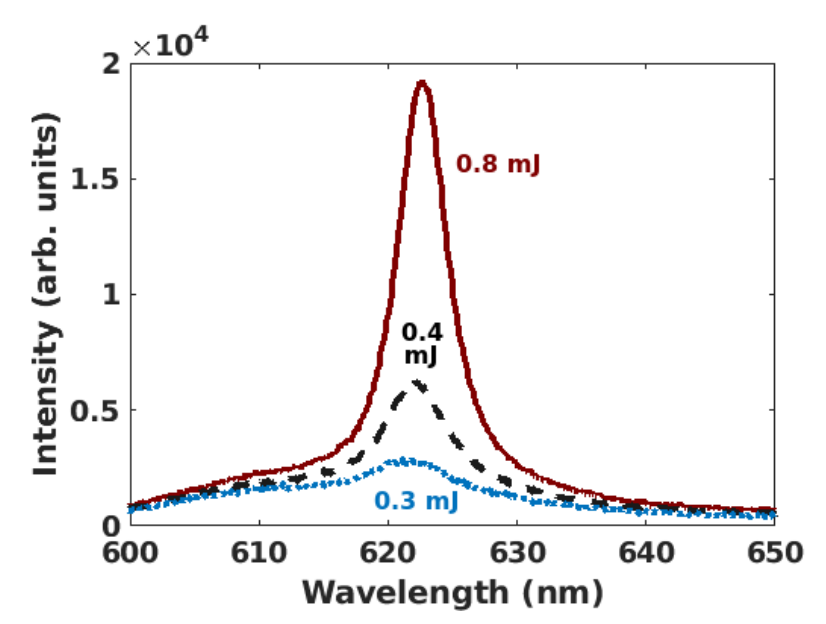

Figure 2. Examples of signal from a sample with particles of $190 \mathrm{~nm}$ of diameter $\left(\mu_{s}^{\prime}=2.33 \mathrm{~mm}^{-1}\right)$ at three different pump energy.

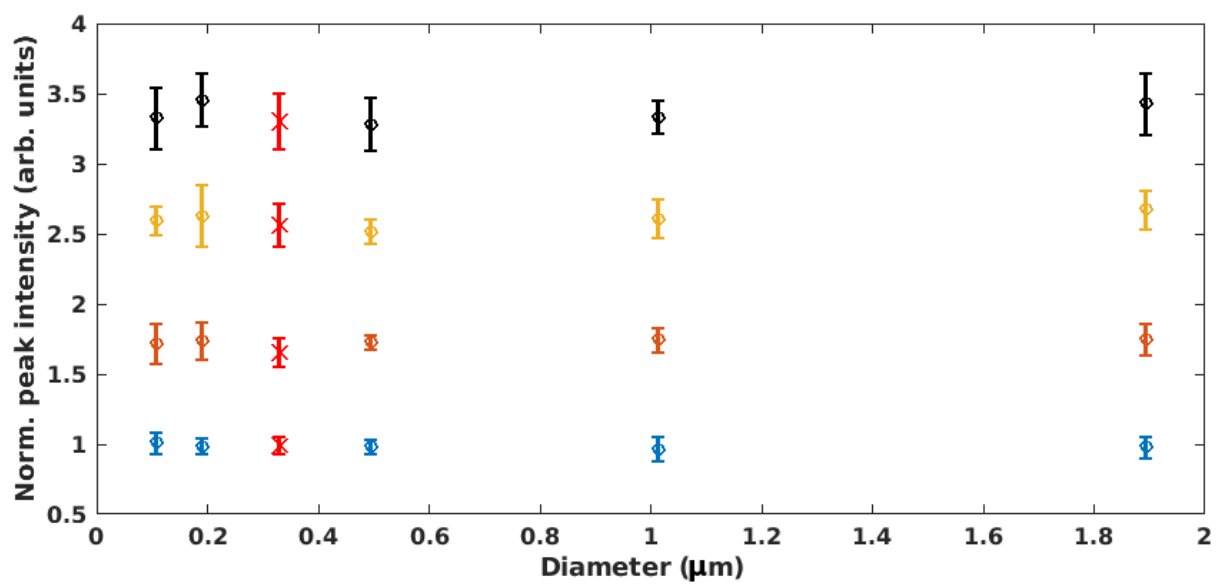

Figure 3. Peak intensity of the signal (normalized to the water value) for the same pump energy of $1.39 \mathrm{~mJ}$. The red cross is the signal from the Intralipid $20 \%$ with the same $\mu_{s}^{\prime}$.

characteristics of the Mie theory. Such a theory allows to determine $g$ and $Q$ (the scattering efficiency) of a single particle. The reduced scattering coefficient $\mu_{s}^{\prime}$, for a monodispersion of spherical particles of radius $r$, is given by: ${ }^{42}$

$$
\mu_{s}^{\prime}=\frac{3}{4} \rho_{v} \frac{Q(1-g)}{r}
$$

where $\rho_{v}$ is the fractional volume of the particles. $Q$ and $g$ are in general complicated functions of the relative refractive index of the scatterer $n_{r}$ and the size parameter $x=2 \pi r / \lambda_{b}$, where $\lambda_{b}$ is the radiation wavelength in the bulk material. To obtain the desired $\mu_{s}^{\prime}$, the concentration of each sample, achieved by adding pure water, has been fixed according to Eq. 1, given the size of the particle and the refractive index of the material, once the single particle coefficients $Q$ and $g$ are calculated by a computer program based on Mie theory. ${ }^{43}$

In order to increase the accuracy in determining the sample concentration, the deviation of $Q$ and $g$ upon the different wavelengths of the dye emission spectrum and upon the size distribution of the particles radius must be considered. For the sizes of scattering particles we assumed a lognormal distribution $f(r)$ of the particles radius, 


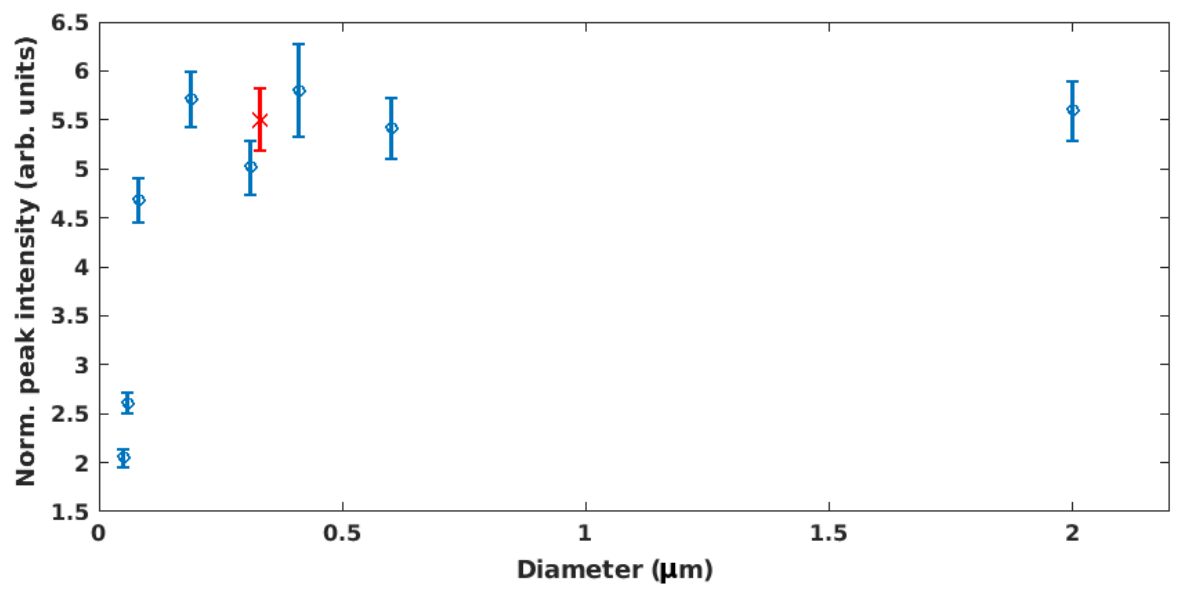

Figure 4. Peak intensity of the signal (normalized to the water value) for the same pump energy at different pump energies. The red crosses are the signal from the Intralipid $20 \%$ with the same $\mu_{s}^{\prime}$.

with the mean value $\bar{r}$ and standard deviation $\sigma$ provided by the manufacturer:

$$
f(r)=\frac{1}{r S \sqrt{2 \pi}} \exp \left[-\frac{1}{2}\left(\frac{\ln r-m}{S^{2}}\right)^{2}\right]
$$

where:

$$
\begin{aligned}
& m=\ln \left(\frac{\bar{r}^{2}}{\sqrt{\sigma^{2}+\bar{r}^{2}}}\right) \\
& S=\sqrt{\ln \left(\frac{\sigma^{2}}{\bar{r}^{2}}+1\right)}
\end{aligned}
$$

Fixed the wavelength, the single scattering parameters $Q$ and $g$ are obtained by averaging over the sphere radius distribution:

$$
\begin{gathered}
Q(\lambda)=\int_{0}^{\infty} d r f(r) Q(r, \lambda) \\
g(\lambda)=\int_{0}^{\infty} d r f(r) g(r, \lambda)
\end{gathered}
$$

The spectral analysis of the signal from the random laser sensor becomes the a posteriori measurement to determine the scattering property of the samples.

\section{RESULTS}

The measurements have been carried out using two different sensors and two sets of particles of different manufacturers.

In Fig. 2 we report typical spectra for the random laser signal, for three different pump energies, from a scattering sample composed by a water dispersion of particles of $190 \mathrm{~nm}$ diameter.

In Fig. 3 the peak intensity, normalized to the water value, is shown as a function of particles diameter at different pump energies $(0.19 \mathrm{~mJ}, 0.39 \mathrm{~mJ}, 0.65 \mathrm{~mJ}$ and $0.89 \mathrm{~mJ})$. The peak intensity is independent of the diameter in the range analyzed (from $\approx 100 \mathrm{~nm}$ to $\approx 2 \mu \mathrm{m}$ ). Moreover, the peak intensity is consistent to that of Intralipid $20 \%$ with the same $\mu_{s}^{\prime}$ (red crosses at the nominal diameter of $0.33 \mu \mathrm{m}$ ). These results indicate that, by previous calibration of the sensor with a reference medium (Intralipid20\%), the $\mu_{s}^{\prime}$ of a material is directly measurable by a fast and simple procedure. In Fig. 4, another set of particles are sensed with a second sensor that is characterized by a stronger signal. The peak value is consistent with the one from Intralipid $20 \%$ for particles with diameter greater than $100 \mathrm{~nm}$. 


\section{CONCLUSIONS}

The results show that the peak of the random laser signal from our sensor only depends on the reduced scattering $\mu_{s}^{\prime}$ of the sensed external medium for particles of a diameter grater than $\sim 100 \mathrm{~nm}$. Then, once calibrated by a reference medium, the sensor guaranties a direct measure of the $\mu_{s}^{\prime}$ of the sample for a wide range of particles diameters. The use of different sensors shows the reproducibility of the measurement.

\section{REFERENCES}

[1] Ignesti, E., Tommasi, F., Fini, L., Martelli, F., Azzali, N., and Cavalieri, S., "A new class of optical sensors: a random laser based device," Sci. Rep. 6, 35225 (2016).

[2] Tommasi, F., Ignesti, E., Fini, L., Martelli, F., and Cavalieri, S., "Random laser based method for direct measurement of scattering properties," Opt. Express 26, 27615-27627 (Oct 2018).

[3] Letokhov, V. S., "Generation of light by scattering medium with negative resonance absorption," Eksp. Teor. Fiz. 53, 1442 (1967).

[4] Wiersma, D. S., "The physics and applications of random lasers," Nat. Phys. 4, 359 (2008).

[5] Wiersma, D. S. and Lagendijk, A., "Light diffusion with gain and random lasers," Phys. Rev. E 54, 4256 (1996).

[6] Mujumdar, S., Ricci, M., Torre, R., and Wiersma, D. S., "Amplified extended modes in random lasers," Phys. Rev. Lett. 93, 053903 (2004).

[7] Mujumdar, S., Tuerk, V., Torre, R., and Wiersma, D. S., "Chaotic behavior of a random laser with static disorder," Phys. Rev. A 76, 033807 (2007).

[8] Lepri, S., Cavalieri, S., Oppo, G. L., and Wiersma, D. S., "Statistical regimes of random laser fluctuations," Phys. Rev. A 75, 063820 (2007).

[9] Ignesti, E., Tommasi, F., Fini, L., Lepri, S., Radhalakshmi, V., Wiersma, D. S., and Cavalieri, S., "Experimental and theoretical investigation of statistical regimes in random laser emission," Phys. Rev. A 88, 033820 (2013).

[10] Tommasi, F., Ignesti, E., Fini, L., and Cavalieri, S., "Controlling directionality and the statistical regime of the random laser emission," Phys. Rev. A 91, 033820 (2015).

[11] Tommasi, F., Fini, L., Ignesti, E., Lepri, S., Martelli, F., and Cavalieri, S., "Statistical outliers in random laser emission," Phys. Rev. A 98, 053816 (Nov 2018).

[12] Gofraniha, N., Viola, I., Di Maria, F., Barbarella, G., Gigli, G., Leuzzi, L., and Conti, C. Nat. Commun. 6, 6058 (2015).

[13] Tommasi, F., Ignesti, E., Lepri, S., and Cavalieri, S., "Robustness of replica symmetry breaking phenomenology in random laser," Sci. Rep. 6, 37113 (2016).

[14] Biswas, S. and Kumbhakar, P., "Continuous wave random lasing in naturally occurring biocompatible pigments and reduction of lasing threshold using triangular silver nanostructures as scattering media," Nanoscale 9, 18812-18818 (2017).

[15] Lawandy, N. M., Balachandran, R. M., Gomes, A. S. L., and Suvain, E., "Laser action in strongly scattering media," Nature 368, 436 (1995).

[16] Cao, H., Zhao, Y. G., Ho, S. T., Seelig, E. W., Wang, Q. H., and Chang, R. P. H., "Random laser action in semiconductor powder," Phys. Rev. Lett. 82(11), 2278 (1999).

[17] Wang, M., Liu, D., He, N., and S L Jacques, S. L. T., "Biological laser action," Appl. Opt. 35(10)(10), 1775 - 1779 (1996).

[18] Polson, R. C. and Vardeny, Z. V., "Organic random lasers in the weak-scattering regime," Phys. Rev. B 71, 045205 (Jan 2005).

[19] Zhang, D., Kostovski, G., Karnutsch, C., and Mitchell, A., "Random lasing from dye doped polymer within biological source scatters: The pomponia imperatorial cicada wing random nanostructures," Org. Electron. 13(11)(11), 2342 - 2345 (2012).

[20] Wang, C.-S., Chang, T., Lin, T.-Y., and Chen, Y.-F., "Biologically inspired flexible quasi-single-mode random laser: An integration of pieris canidia butterfly wing and semiconductors," Sci. Rep. 4, 6736 (2014).

[21] Humar, M. and Yun, S. H., "Intracellular microlasers," Nat. Photonics 9, 572-576 (2015). 
[22] Schubert, M., Steude, A., Liehm, P., Kronenberg, N. M., Karl, M., Campbell, E. C., Powis, S. J., and Gather, M., "Lasing within live cells containing intracellular optical microresonators for barcode-type cell tagging and tracking," Nano Lett. 15(8), 5647-52 (2015).

[23] Hohmann, M., Dörner, D., Mehari, F., Chen, C., Späth, M., Müller, S., Albrecht, H., Klämpfl, F., and Schmidt, M., "Investigation of random lasing as a feedback mechanism for tissue differentiation during laser surgery," Biomed. Opt. Express 10, 807-816 (Feb 2019).

[24] Polson, R. C. and Vardeny, Z. V., "Random lasing in human tissues," Appl. Phys. Lett. 85, 1289 (2004).

[25] Durduran, T., Choe, R., Baker, W. B., and Yodh, A. G., "Diffuse optics for tissue monitoring and tomography," Reports on Progress in Physics 73(7), 076701 (2010).

[26] Wang, Y., Duan, Z., Qiu, Z., Zhang, P., Wu, J., Zhang, D., and Xiang, T., "Random lasing in human tissues embedded with organic dyes for cancer diagnosis," Sci. Rep. 7, 8385 (2017).

[27] Lahoz, F., Martìn, I. R., Urgellís, M., Marrero-Alonso, J., Marìn, R., Saavedra, C. J., Boto, A., and Dìaz, M., "Random laser in biological tissues impregnated with a fluorescent anticancer drug," Laser Physics Letters 12(4), 045805 (2015).

[28] Lahoz, F., Martín, I. R., Urgellés, M., Marrero-Alonso, J., Marín, R., Saavedra, C. J., Boto, A., and Díaz, M., "Random laser in biological tissues impregnated with a fluorescent anticancer drug," Laser Physics Letters 12, 045805 (mar 2015).

[29] Cao, H., "Random lasers: Development, features and applications," Opt. Photon. News 16, $24-29$ (Jan 2005).

[30] Choi, S. H. and Kim, Y. L., "The potential of naturally occurring lasing for biological and chemical sensors," Biomed. Eng. Lett. 4(3), 201-212 (Sep 2014).

[31] Xu, Y., Zhang, L., Gao, S., Lu, P., Mihailov, S., and Bao, X., "Highly sensitive fiber random-grating-based random laser sensor for ultrasound detection," Opt. Lett. 42(7), 1353-1356 (Apr 2017).

[32] Gaio, M., Caixeiro, S., Marelli, B., Omenetto, F. G., and Sapienza, R., "Gain-based mechanism for $p \mathrm{H}$ sensing based on random lasing," Phys. Rev. Applied 7, 034005 (Mar 2017).

[33] Caixeiro, S., Gaio, M., Marelli, B., Omenetto, F. G., and Sapienza, R., "Silk-based biocompatible random lasing," Advanced Optical Materials 4(7), 998-1003 (2016).

[34] Song, Q., Xiao, S., Xu, Z., Liu, J., Sun, X., Drachev, V., Shalaev, V. M., Akkus, O., and Kim, Y. L., "Random lasing in bone tissue," Opt. Lett. 35(9), 1425-1427 (May 2010).

[35] van Staveren, H. J., Moes, C. J. M., van Marie, J., Prahl, S. A., and van Gemert, M. J. C., "Light scattering in lntralipid-10\% in the wavelength range of 400-1100 nm," Appl. Opt. 30(31), 4507-4514 (Nov 1991).

[36] Michels, R., Foschum, F., and Kienle, A., "Optical properties of fat emulsions," Opt. Express 16, 5907-5925 (Apr 2008).

[37] Di Ninni, P., Martelli, F., and Zaccanti, G., "Intralipid: towards a diffusive reference standard for optical tissue phantoms," Phys. Med. Biol. 56(2)(2), N21 (2011).

[38] Martelli, F. and Zaccanti, G., "Calibration of scattering and absorption properties of a liquid diffusive medium at nir wavelengths. cw method," Opt. Express 15(2), 486-500 (Jan 2007).

[39] Zaccanti, G., Del Bianco, S., and Martelli, F., "Measurements of optical properties of high-density media," Appl. Opt. 42, 4023-4030 (Jul 2003).

[40] Di Ninni, P., Bérubé-Lauzière, Y., Mercatelli, L., Sani, E., and Martelli, F., "Fat emulsions as diffusive reference standards for tissue simulating phantoms?," Appl. Opt. 51(30), 7176-7182 (Oct 2012).

[41] Aernouts, B., Zamora-Rojas, E., Beers, R. V., Watté, R., Wang, L., Tsuta, M., Lammertyn, J., and Saeys, W., "Supercontinuum laser based optical characterization of intralipid phantoms in the 500-2250 nm range," Opt. Express 21(26), 32450-32467 (Dec 2013).

[42] Martelli, F., Del Bianco, S., Ismaelli, A., and Zaccanti, G., [Light Propagation through Biological Tissue and other Diffusive Media], SPIE Press/Bellingham, Whashington (USA) (2009).

[43] Bohren, C. F. and Huffman, D., [Absorption and scattering of light by small particles], Wiley science paperback series, Wiley (1983). 